\title{
Dirk Messner
}

\section{Ist Außenpolitik noch Außenpolitik ... und was ist eigentlich Innenpolitik? Die Transformation der Politik in der "Ära des Globalismus"}

Sicher ist, daß Globalisierungsprozesse das Koordinatensystem der Politik nachhaltig verändern - die „Epoche der Nationalstaaten“, die am 24. Oktober 1648 mit dem „Westfälischen Frieden“ begann, neigt sich ihrem Ende zu. Unsicher und allenfalls schemenhaft umschreibbar ist jedoch, welches neue Koordinatensystem der Politik in der „Ära des Globalismus“ (Kaiser/ May 1995: 396) entsteht. Der Versuch, die Transformationen der Politik - die Zukunft der Nationalstaaten, der Demokratie, der internationalen, transnationalen und globalen Politik, einer entstehenden Global Governance-Architektur - in diesem Umbruch adäquat zu erfassen und daraus handlungsorientierte Schlußfolgerungen $\mathrm{zu}$ ziehen, ist bei weitem nicht abgeschlossen. Schon die Tatsache, daß dem Nationalstaat im Kontext der Globalisierung je nach Beobachtungsperspektive sein schleichendes Ende (Guéhenno 1995, Ohmae 1995), robuste Gesundheit (Mann 1997), gar gesteigerte Handlungsfähigkeit (Milward 1992) oder auch strukturell begrenzte, aber seit Jahrzehnten stabile Gestaltungs(un)fähigkeit (Narr 1999) bescheinigt wird, reflektiert den offenen Such- und Lemprozeß der Sozialwissenschaften in diesem für sie zentralen Feld. Vor dem Hintergrund vieler offener Fragen sollen im folgenden vier Kernelemente des Wandels der Architektur der Politik diskutiert werden: (1) die rasante Ausdifferenzierung der Außenbeziehungen der Nationalstaaten als Indikator der Erosion der klassischen Grenzen von Innen- und Außenpolitik; (2) der Trend zur Herausbildung einer Weltgesellschaft; (3) die Verdichtung grenzüberschreitender Vernetzungen und globaler Problemlagen, die nicht nur zu einer Zunahme zwischenstaatlicher Interdependenzbeziehungen (eines lange bekannten Phänomens), sondern zu einer Erosion „interner Souveränität" der Nationalstaaten führen, welche die zukünftigen Spielregeln internationaler und globaler Politik auf den Kopf stellen; (4) der Formwandel politischer Macht unter den Bedingungen der Globalisierung. 


\section{Ist Außenpolitik noch Außenpolitik? Trends in der Außenpolitik aus nationalstaatlicher Perspektive}

Die vergangenen drei Jahrhunderte waren gekennzeichnet durch eine enorme Ausdifferenzierung der Aufgabenfelder und des institutionellen Designs des Nationalstaates zur Gestaltung der „Innenpolitik“. Seit dem 18. Jahrhundert dehnten sich die staatlichen Bürokratien sukzessive auf alle gesellschaftlichen Lebensbereiche aus. Die Herstellung und Sicherung grundlegender öffentlicher Güter wie innere Sicherheit, Rechtssicherheit, Geld und Infrastruktur standen am Anfang dieses Prozesses. In den Wohlfahrtsstaaten der zweiten Hälfte des 20. Jahrhunderts intervenieren die Nationalstaaten in die Verteilung von Einkommen, sichern Beschäftigung, verwalten soziale Sicherungssysteme, produzieren in öffentlichen Unternehmen einen beachtlichen Teil des Bruttosozialproduktes, regeln Geschlechterverhältnisse, managen das $\mathrm{Ge}-$ sundheitswesen und spielen eine zentrale Rolle in den Bildungs-, Universitätsund Forschungslandschaften. Der Prozeß der Ausdifferenzierung der innenpolitischen Institutionensysteme und erfolgreiche Demokratisierung gehen einher mit der Steigerung gesellschaftlicher Handlungsfähigkeit, so daß die Nationalstaaten trotz, oder besser aufgrund ihrer wachsenden Interventionen in Gesellschaft und Wirtschaft ihre Rolle als einheitliches Zentrum und Spitze des kollektiven Gemeinwesens sukzessive verlieren. Entgegen den Idealtypen der Pluralismustheorie werden die klaren Grenzen zwischen öffentlichen und privaten Akteuren porös. Politik findet nun zunehmend in sektoralen Verhandlungssystemen statt, die auf Netzwerkstrukturen basieren, in denen staatliche und private Organisationen zusammenwirken (Kenis/Schneider 1991; Scharpf/Mayntz 1995; Messner 1998). Die Ausdehnung der Staatstätigkeiten resultierte in den westlichen Sozialstaaten also nicht etwa in der Etablierung von „strong states and weak societies“, sondern in Modernisierungsprozessen, die zu dichten institutionellen Strukturen (Streeck 1991: 27) führen, die die Rationalität der Teilsysteme erhöhen, wodurch jedoch zugleich auch die Irrationalität des Ganzen gesteigert werden kann. Es sind diese gesellschaftlichen Strukturveränderungen die den Übergang von der keynesianischen Globalsteuerungsphilosophie der 60er und 70er Jahre zu Netzwerkgesellschaften markieren, in denen sich markt-, hierarchie- und netzwerkbasierte Steuerungsmuster ergänzen (Messner 1998: 72ff.).

\section{Die institutionelle Transformation der Außenpolitik: die Binnenperspektive}

Die Außenpolitik bleibt von der Dynamik der Komplexitätssteigerung der Innenpolitik zunächst scheinbar unberührt. Zwar entsteht im Verlauf der 50er und 60er Jahre eine Vielzahl Internationaler Organisationen, deren Gesamtzahl sich auf etwa 3000 summiert, und seit den 70er Jahren gewinnen Internationale Regime an Bedeutung, so daß sich eine gewisse Akteursvielfalt 
entwickelt (Beisheim et al. 1999: 325 ff.). Das Politikfeld „Außenpolitik“ bleibt jedoch übersichtlich: Die Nationalstaaten sind die entscheidenden Akteure der internationalen Politik, in deren Zentrum aus der Sicht der jeweiligen Staaten Friedenssicherung und Verteidigung, die Marktöffnung für eigene Exporte und - als eher randständiges Feld - die Entwicklungszusammenarbeit stehen. Vor diesem Hintergrund lassen sich auch Innen- und Außenpolitik ziemlich trennscharf unterscheiden.

Dieses überschaubare Panorama dürfte grundlegend dafür sein, daß in Deutschland, wie ein Blick in die Geschäftsordnung der Bundesregierung verrät, das Auswärtige Amt eine umfassende Verantwortung für die Gesamtheit der deutschen Außenbeziehungen und die Wahrnehmung ,gesamtstaatlicher Interessen" hat, flankiert durch das Bundeskanzleramt, das aufgrund der Richtlinienkompetenz des Kanzlers auch in der Außenpolitik eine starke Stellung besitzt. So können Verhandlungen mit dem Ausland oder im Ausland nur mit Zustimmung des Auswärtigen Amtes, auf Verlangen auch nur unter seiner Mitwirkung geführt werden ( $\$ 11$ der Geschäftsordnung). Während in der Innenpolitik die immer weitere Auffächerung von Politikbereichen, institutionelle Vielfalt sowie die steigende Komplexität von Problemlagen in der realen Politik und in der Wissenschaft dazu beigetragen haben, von hierarchischen Steuerungskonzepten („des Planungsstaates“, der „Globalsteuerung“ usw.) abzurücken und die Rolle nationalstaatlicher Institutionen im gesamtgesellschaftlichen Beziehungsgeflecht zu relativieren, ist im Feld der Außenpolitik die Illusion der hierarchischen Steuerung durch einen zentralen Akteur, der beansprucht die „nationalstaatlichen Gesamtinteressen“ zu vertreten, noch am Anfang des 21. Jahrhunderts weit verbreitet.

$\mathrm{Daß}$ es sich um eine Illusion handelt, wird rasch deutlich, wenn man sich, um bei dem deutschen Beispiel zu bleiben, einen Überblick über die nationalen Institutionen verschafft, die tatsächlich Außenbeziehungen betreiben. Das Bild, das entsteht, veranschaulicht, daß in den vergangenen, vielleicht zwei Dekaden im Zusammenhang mit ökonomischen, technologischen und kulturellen Globalisierungsprozessen, der europäischen Integration sowie der zunehmenden Wahrnehmung von Welt- und Menschheitsproblemen als zentralen Herausforderungen des 21. Jahrhunderts (Messner/Nuscheler 1999) nun auch die „Außenpolitik“ einen enormen institutionellen Ausdifferenzierungsprozeß erlebt vergleichbar der Entwicklungsdynamik in der Innenpolitik in den vergangenen Jahrhunderten. Außenpolitische Entscheidungsprozesse sind in der realen Welt der „Epoche des Globalismus“ längst nicht mehr die exklusive Domäne der klassischen Außen- und Sicherheitspolitik, mit dem Außenministerium als steuerndem Zentrum. Verblüffend ist dabei weniger diese Entwicklung an sich, sondern vielmehr die Tatsache, daß sie sich weitgehend unbemerkt und kaum problematisiert von der Wissenschaft vollzogen hat. Die institutionelle Ausdifferenzierung der Außenpolitik wird durch die folgenden Entwicklungen sichtbar: 
250 Referate oder vergleichbare Arbeitseinheiten anderer Ressorts (jenseits des Auswärtigen Amtes, des Verteidigungs- und des Entwicklungsministeriums) sind derzeit mit europa- und außenpolitischen Fragen befaßt. Das Auswärtige Amt verfügt seit 1998 noch über 68 vergleichbare Arbeitseinheiten! Im Ergebnis ist damit faktisch jedes Fachministerium zum „Außenministerium“ des von ihm bearbeiteten Politikfeldes geworden. Umwelt-, Sozial-, Wirtschafts- und Finanz-, Technologie- oder auch Energiepolitiken wachsen immer weiter in den internationalen und globalen Raum hinein. Selbst das Innenministerium, das die Abgrenzung zur Außenpolitik in seinem Namen trägt, beschäftigt sich zunehmend mit grenzüberschreitenden Fragen, wie Migration, Kriegsflüchtlingen, internationaler Kriminalität sowie Asylpolitik und unterhält in diesen Feldern enger Arbeitskontakte mit korrespondierenden Bürokratien anderer Länder und Internationalen Organisationen (SiwertProbst 1998, Andreae/Kaiser 1998). Etwa die Hälfte der Beamten des höheren Dienstes aller Ministerien sind kontinuierlich in politische Entscheidungen im Rahmen der Europäischen Union, also in grenzüberschreitende Beziehungsnetzwerke, eingebunden (Eberwein/Kaiser 1998). Von 11,8 Mrd. DMark, die der Bund 1996 für Auswärtige Angelegenheiten ausgab, entfielen nur knapp ein Drittel auf das Auswärtige Amt. Auch die Bundesländer haben ihre grenzüberschreitenden Aktivitäten in den vergangenen Dekaden weiter ausgebaut und sind $\mathrm{zu}$ mehr oder weniger autonomen Akteuren deutscher Außenpolitik geworden.

Hinzu kommt, daß NGOs, aber auch Unternehmen und deren Organisationen in der Außenpolitik zu einem wichtigeren Einflußfaktor geworden sind, also öffentliche Institutionen zunehmend Konkurrenz von privaten Akteuren der „Wirtschafts- und Gesellschaftswelt“ (Czempiel 1993 und 1999) bekommen. Der Einfluß von NGOs im Rahmen der Weltkonferenzen der 80er und 90er Jahre, die Präsenz von NGOs im Umfeld des WTO-Gipfels von Seattle oder auch die Lobby der (seit einiger Zeit erodierenden) Anti-KlimapolitikAllianz großer Energie- und Erdölunternehmen, sind Beispiele dafür, daß Außenpolitik nicht mehr nur eine Sache der Staatenwelt ist (Altvater et al. 1997, Methews 1997, Messner 1999a).

Es zeigt sich erstens, daß alle Ministerien in den internationalen Raum hineingewachsen sind und sich dadurch die lange Zeit zentrale Stellung klassischer Außen- und Sicherheitspolitik und der hierfür zuständigen Ministerien für die Gestaltung der Außenbeziehungen relativiert hat. Ausdifferenzierung und Enthierarchisierung sind Merkmale der Transformation der Außenpolitik in der Phase des Übergangs von der „Epoche der Nationalstaaten“ zur „Ära der Globalismus“. Die Ausdifferenzierungsprozesse implizieren zweitens auch die Perforierung von Grenzen zwischen dem öffentlichen und dem privaten Sektor. Ähnlich wie in der Innenpolitik in den Dekaden zuvor, werden nun gesellschaftliche Akteure zu aktiven Mitspielern im außenpolitischen Feld 
(Methews 1997). ${ }^{1}$ Deutlich wird drittens, daß „reine Außenpolitik“ unter den Bedingungen von Globalisierung immer seltener wird - ebenso wie „reine“ Innenpolitik. Innen- und Außenpolitik werden immer enger miteinander verwoben. Die wechselseitige Durchdringung der nationalen staatlichen Institutionen und ihres gesellschaftlichen Umfeldes mit der internationalen und globalen Umwelt verbindet die ehemals getrennt Welten der Innen- und Außenpolitik zu ,intermestiv affairs“ (Manning 1977). ${ }^{2}$

\section{Die Transformation der Internationalen Beziehungen Von der Staatenwelt zur Weltgesellschaft?}

Die skizzierten Ausdifferenzierungstrends in den außenpolitischen Institutionen der Nationalstaaten korrespondieren mit immer dichteren internationalen und globalen institutionellen Strukturen, die die Dynamik der Enthierarchisierung von Außenpolitik sowie die Entgrenzung von Innen- und Außenpolitik zusätzlich verstärken (Messner 1999a). Etwa 3000 Internationale Organisationen, in denen sich sukzessive globale Sichtweisen herausbilden können, die nicht mehr unmittelbar an nationale Interessen rückgebunden sind, bilden Grundbausteine der Epoche des Globalismus.

Die Zahl der global agierenden NGOs wird auf gut 10.000 geschätzt. Sie bilden den Humus einer globalen Zivilgesellschaft. Auch die ca. 45.000 multinationalen Unternehmen und deren Interessenorganisationen werden zu bedeutenden Akteuren auf der globalen Bühne. Die zunehmend global organisierte Wissenschaft, die universalistisch-kosmopolitischen Traditionen folgt, spielt im Rahmen der Global Governance-Architektur eine wichtige Rolle. Von großer Bedeutung ist nicht zuletzt der sukzessive Übergang von einem „Zwischenstaatlichen Völkerrecht“ hin zu einer Ordnung, in der Elemente eines Weltinnenrechtes entstehen (Delbrück 1998). Schließlich sind Medien, Kommunikations- und Informationstechnologien wesentliche Katalysatoren

1 Haass (1999: 38) bringt diesen durchaus ambivalenten Sachverhalt auf den Punkt: „,nonstate actors ... ranging from Usama bin Ladin to Amnesty International to the International Criminal Court to George Soros ... are increasingly in number and acquiring power."

2 Aufgrund der skizzierten Strukturen spreche ich/wir (Messner/Nuscheler) von „Politik in der Global Governance-Architektur“ und damit von Politik auf der lokalen, nationalen, regionalen und globalen Ebene. Der globale Raum wird zum Referenzrahmen für Politik (ähnlich wie die Weltwirtschaft zum Referenzrahmen ökonomischer Akteure wird), was nicht heißt, daß alle Politik globale Dimensionen annehmen muß (genauso wie nicht alle Unternehmen in globale Beziehungsgeflechte eingebunden sind). Politik in der Global Governance-Architektur darf daher nicht auf „Politik auf der globalen Ebene“ reduziert werden. Angesichts der zunehmenden Entgrenzung von Innen- und Außenpolitik müßte man eigentlich präzise zwischen den Ebenen unterscheiden, auf denen Akteure handeln, zugleich die Ebenen benennen, auf die ihr Handeln (intendiert oder nicht intendiert) einwirkt oder einwirken soll und gegebenenfalls die Verknüpfung mit weiteren Handlungsebenen berücksichtigen. Im folgenden wird in Ermangelung einer einfachen Terminologie weiterhin von „Innenund Außenpolitik" gesprochen, aber das Zusammenwirken von unterschiedlichen Handlungsebenen in der Global Governance-Architektur berücksichtigt. 
einer immer engeren Vernetzung lokaler, nationaler, regionaler und globaler Handlungsebenen und Akteure. In diesem Kontext multiplizieren sich grenzüberschreitende Interaktionen und Vernetzung von politischen, ökonomischen und sozialen Handlungsebenen.

Vor diesem Panorama gibt es gute Gründe für das Argument, daß die Globalisierung einen Prozeß in Richtung der Herausbildung einer Weltgesellschaft befördert. John Burton hatte das bis heute umstrittene Konzept der „Weltgesellschaft“ bereits 1972 in die Diskussion gebracht (zur Kritik: Altvater/Mahnkopf 1996). Versteht man unter „Gesellschaft“ das „umfassendste System menschlichen Zusammenlebens“ oder das „Sozialsystem höchster Ordnung“ (Talcott Parsons), dann ist es folgerichtig, im engeren Sinne von nationalen Gesellschaften und im weiteren Sinne von der sich entwickelnden Weltgesellschaft zu sprechen. Die grenzüberschreitende Dynamik vieler gesellschaftlicher Funktionssysteme (z.B. Wirtschaft, Umwelt, Recht, Wissenschaft, Militär), interund transnationale Interdependenzen, die miteinander verwobene Mehrebenenpolitik von der Lokal- bis zur Globalpolitik, die weltweite Vereinheitlichung von Standards, Normen und Regulationsmustern, regionale Integrationsprozesse sowie die Herausbildung global agierender Akteure (Konzerne wie Daimler-Chrysler; NGOs wie Greenpeace) führen zu Strukturen, die mit dem Begriff des ,internationalen Systems“ nicht mehr hinreichend abgebildet werden können.

Ernst-Otto Czempiel (1993: 106f.) ist daher zuzustimmen, wenn er schreibt, daß die Welt „noch keine Weltgesellschaft, aber auch keine Staatenwelt mehr ist“. Die Verdichtung globaler Trends führt jedoch schrittweise zur Herausbildung einer Weltgesellschaft - diese wird eine soziale Ordnung (eine Gesellschaft) sein, die sich qualitativ von den nationalen Gesellschaften unterscheidet (genauer: Forschungsgruppe Weltgesellschaft 1996, Messner 1999b). In ihr gibt es weiterhin Alternativen, z.B. hinsichtlich der Ausgestaltung konkreter Produktionsverhältnisse, von kulturellen Mustern, von Sozial- und Umweltregulierungssystemen sowie von Lebensweisen. Auch die nationalen Gesellschaften verschwinden nicht, werden aber in immer dichtere Beziehungsnetzwerke der entstehenden Weltgesellschaft eingewoben und verändern sich in diesem Prozeß ebenfalls.

\section{Das Koordinatensystem und die Architektur der Politik im Umbruch}

Die Wirkungen der beschriebenen Entwicklungsdynamiken auf die Architektur der Politik (polity) und die Dynamik politischer Prozesse (policy und politics) im Kontext der Globalisierung können im Rahmen eines Aufsatzes nicht annähernd befriedigend thematisiert werden. Dennoch sollen einige Elemente skizziert und Fragen aufgeworfen werden, die die Richtung der 
Transformation der Politik und die Verschiebungen zwischen nationalstaatlicher und globaler Politik thematisieren.

\section{Das nationale System der Außenpolitik unter Anpassungsdruck}

Aus der bereits beschriebenen Transformation der Außenpolitik ergibt sich zunächst, daß diese nicht mehr aus „einem Guß“ und aus „einer Hand“ sein kann. Daraus entsteht eine Vielzahl von institutionellen Problemen und Herausforderungen, um trotz Akteursvielfalt zu einer möglichst kohärenten Außenpolitik zu kommen. Zu diesem Fragenkomplex gehört die Suche nach der zukünftigen Rolle des Außenministeriums, als einer unter vielen Institutionen, die für deutsche Außenbeziehungen zuständig ist, genauso, wie die steuerungstheoretisch alles andere als triviale Frage nach tragfähigen Mustern der Koordination zwischen den beteiligten Akteuren und den notwendigen intra- und interorganisatorischen Reformen der Ministerienlandschaft. Interessanterweise sind diese institutionellen Herausforderungen, die sich aus Globalisierungsprozessen ergeben, bisher kaum untersucht. Sie spielen auch in den politischen Debatten über „Antworten auf die Globalisierung“ kaum eine Rolle, die sich, wie auch eine Auswertung der Ergebnisse der Weltkonferenzen der vergangenen Jahre zeigte (Messner/Nuscheler 1996), im wesentlichen auf policy-Dimensionen konzentrieren. Wissenschaft und Politik sollten sich intensiver um die Institutionen der Politik und deren Umbau kümmern, da ansonsten eine tragfähige „Wiedereinbettung“ der Eigendynamiken der Globalisierung unmöglich wird. Derzeit entwickeln sich nationale Gesellschaften und ihre Institutionen in Richtung auf eine „Gesellschaft der zwei Geschwindigkeiten“. Die unter hohem internationalen Wettbewerbsdruck stehenden Unternehmen (sowie Teilbereiche ihres institutionellen Umfeldes) müssen sich kontinuierlich modernisieren, reorganisieren und den globalen Effizienzstandards anpassen; die öffentlichen Einrichtungen und das politische System sind aus vielfältigen Gründen durch ein hohes Maß an institutionellem Strukturkonservatismus geprägt. Dieses Muster trägt auf Dauer zu einer kontinuierlichen Schwächung öffentlicher Akteure gegenüber der Wirtschaft und damit einer schleichenden Erosion des Primates der Politik (sowie der Säulen des „Rheinischen Kapitalismus“) bei - wenn nicht gegengesteuert wird.

Die Dynamik der Transformation der Außenpolitik unterstreicht darüber hinaus, daß für nahezu jedes Politikfeld gilt, daß ohne ein kluges Management der jeweiligen außenpolitischer Interdependenzen, innenpolitische Handlungsspielräume sinken. Innen- und Außenpolitik müssen immer enger miteinander verzahnt und abgestimmt werden. Dies gilt insbesondere im Kontext der Europäischen Integration, in der - neben und quer zu den Nationalstaaten - im europäischen Mehrebenensystem so etwas wie eine „europäische Innenpolitik“ entsteht (Héritier/Knill/Mingers 1996). 
Bedeutsam ist zudem, daß die institutionellen Strukturen, in denen politische Entscheidungsprozesse ablaufen, immer häufiger quer zu den territorialen Grenzen liegen. Um es holzschnittartig ausdrücken: In der Epoche der Nationalstaaten agierte die große Mehrzahl der Akteure - nämlich all diejenigen, die nicht in der Außenpolitik engagiert waren - im nationalen Referenzrahmen. In der Epoche des Globalismus bilden sich zunehmend multi- und transnationale Netzwerke heraus, die die Logik nationalstaatlicher Politik nachhaltig verändern. Letztere zeichnen sich dadurch aus, daß nahezu alle Fachministerien direkt und permanent mit entsprechenden Ministerien anderer Staaten, EU-Behörden, Internationalen Organisationen sowie in unterschiedlicher Form beteiligten, ebenfalls international agierenden NGOs kommunizieren. So entstehen horizontale und grenzüberschreitende Netzwerke, deren Interaktion, basierend auf fachlicher Solidarität und informellen Kontakten jenseits der klassischen diplomatischen Kanäle, dichter ausfallen können als die Interaktionen in den formal zuständigen öffentlichen Institutionen im nationalen Rahmen (Haas 1992). Im Kontext der Klimakonferenzen läßt sich die Entstehung transnationaler Koalitionen von Vertretern von Umweltministerien und -experten beobachten, in denen Problemlösungen erarbeitet und in die internationalen Verhandlungsprozesse eingespeist werden, die vielfach nicht mit den Prioritäten der nationalen Regierungen übereinstimmen. In der Entwicklungspolitik nutzen nationale Entwicklungsministerien oft die öffentliche Artikulationsfähigkeit transnationaler entwicklungspolitischer Netzwerke und Internationaler Organisationen, um die eigene Regierung von der Wichtigkeit bestimmter Politiken zu überzeugen. Entscheidungsfindungsprozesse verändern sich im Prozeß der ,institutionellen Globalisierung", ebenso wie die Funktionen und Bedeutung von Grenzen und Grenzziehungen.

Auch die Metapher des „nationalen Interesses“ schmilzt unter der Globalisierungssonne dahin. Während die traditionelle Außen- und Sicherheitspolitik überzeugend argumentieren konnte, daß es ein "nationales Gesamtinteresse“ an der Entwicklung friedlicher und partnerschaftlicher Beziehungen zu den Nachbarstaaten und der Sicherung des Friedens in Europa und der Welt gibt, fällt ein solches Ansinnen in anderen Politikfeldern schwer. Die Vorstellungen des deutschen Umweltministeriums zur globalen Klimapolitik unterscheiden sich, völlig unabhängig von der jeweiligen Regierungskonstellation, signifikant von denen des Wirtschaftsministeriums. Und während die einen ihre nationalen und internationalen Bündnispartner in der Community der Umweltforscher und organisationen suchen, bilden die anderen Allianzen mit der Wirtschaftswelt. Und dann sind da noch die Umweltverbände und -forscher, die sich mit dem Umweltministerium und der internationalen Versicherungswirtschaft zu einer informellen Allianz gegen die Klimaerwärmung zusammenschließen, und sich hauptsächlich mit der Phalanx der Energie- und Transportunternehmen und 
traditionellen Wirtschaftspolitikern auseinandersetzen müssen. Die Vorstellung, daß pluralistische Gesellschaften im Inneren vielfältige Interessenkonflikte zu bewältigen haben, jedoch in den Außenbeziehungen ein einheitliches „nationales Interesse“ existiere, ist obsolet (Nye 1999).

\section{Die Umrisse einer Global Governance-Architektur werden sichtbar}

Der Ausdifferenzierung der Außenpolitik und der Erosion der Grenzen von Innen- und Außenpolitik entspricht, daß sich lokale, nationale, internationale und globale Politikarenen immer enger vernetzen - grenzüberschreitende Mehrebenenpolitik wird in vielen Politikfeldern zum Regelfall. Eine polyzentrische Global Governance-Architektur entsteht, die bei weitem nicht auf die Summe der Aktivitäten der Nationalstaaten und ihrer Institutionen reduziert werden kann (Messner/Nuscheler 1997, 1999). Nationalstaaten, subnationale Regionen, globale Regime, Internationale Organisationen, in regionalen Integrationsprojekten zusammengeschlossene Ländergruppen und Global Policy Networks (Benner/Reinicke 1999) bilden Kernelemente der Architektur der Politik im 21. Jahrhundert. Die Nationalstaaten bleiben auch in der Epoche des Globalismus die zentralen Orte der Politik, sie werden jedoch in die sich verdichtenden globalen Ordnungsmuster eingebettet (Rosenau 1997; Messner/ Nuscheler 1997; Messner 1998a; Zürn 1998; Albert/Brock 1999; Hewson/ Sinclair 1999; Young 1999).

Die eingängige und vielzitierte Methapher von der Verwandlung der Wohlfahrts- in „Wettbewerbsstaaten“ im Prozeß der Globalisierung und des Standortwettbewerbes (Hirsch 1995) fängt die Komplexität der längst nicht abgeschlossenen Transformationen der Politik in der entstehenden Global Governance-Architektur nicht hinreichend ein. Prozesse der

- Lokalisierung und Dezentralisierung der Politik, in deren Verlauf subnationale (Mikro-)Regionen ihre Handlungsspielräume erweitern können (Durán/ Dussel 1999; Storper 1997),

- Regionalisierung und Zentralisierung von Politik, die zur Herausbildung handlungsfähiger Staatenverbünde oder neuer Formen der Staatlichkeit führen können (Böhret/Wewer 1993; Stallings 1995; Altvater/Mahnkopf 1996; Albert/Brock 1999),

- vertikalen und horizontale Ausdifferenzierung der Politik im Kontext der Global Governance-Architektur (Hauchler et al. 1999, Young 1999),

- Privatisierung der Politik, auf der einen Seite z.B. durch die Schaffung privater Pensionsfonds, die staatliche Sicherungssysteme ablösen, und auf der anderen Seite durch globale NGO-Netzwerke, die z.B. durch die Etablierung von Labels zur Kennzeichnung sozial- und umweltverträglicher Produkte und Produktionsprozesse zur Herausbildung internationaler Sozial- und Umweltstandards beitragen (private governance) (Werle 1995), 
- Ökonomisierung der Politik, die entstehen, weil die Notwendigkeit, global flottierende und strategisch wichtige Ressourcen zu umwerben, die Logik von Wettbewerb und Effizienz verstärken (Streeck 1998);

- Vergesellschaftung der Politik, z.B. durch die vielfältigen Aktivitäten von NGOs und neuen Formen der Kooperation zwischen Staaten und privaten Akteuren (Altvater et al. 1997; Friedrich Ebert Stiftung 1996),

- Re-Regulierung von Politikfeldern und eine erneute Aufwertung öffentlicher Institutionen, infolge der nicht selten desaströsen Ergebnisse neoliberaler Radikalkuren der 80er und 90er Jahre (Gummet 1996; Worldbank 1998) und nicht zuletzt

- globalen Medialisierung der Politik im Kontext der Revolution der Informations- und Kommunikationstechnologien (Kleinsteuber/Thomaß 1998), überlagern sich und bewirken eine Erosion der Grundstrukturen, die die Politik in der Epoche der Nationalstaaten kennzeichnete.

Die Grundausrichtung der entstehenden Global Governance-Architektur ist keineswegs sicher. Vier Szenarien sind denkbar: Global Governance-Strukturen wären erstens denkbar als Versuch der USA, ihre hegemoniale Rolle als einzig verbliebene Supermacht zu stabilisieren und entsprechend das Design der internationalen und globalen Politik nach ihren Interessen zu ordnen und auszugestalten, gemäß der Devise, daß „Empires have no interest in cooperation within an international system, they aspire to be the international system" (Chace/Rizopoulos 1999: 3). Global Governance könnte zweitens zu einem kooperativen Programm werden, das im wesentlichen die OECD-Welt enger zusammenführt, möglicherweise verknüpft mit der selektiven „Assoziation“ einiger zentraler Schwellenländer, aber unter Ausschluß großer Teile der Entwicklungsländerregionen. Global Governance könnte drittens auf einigen starken, handlungsfähigen regionalen Kernen in Weltwirtschaft und -gesellschaft, also einer engen Vernetzung von Regional- und Global Governance basieren und ist viertens als eine umfassende kooperative Architektur denkbar, die auch Entwicklungsländerregionen aktiv in den Prozeß der globalen Problemlösung miteinbezieht (die Szenarien 2-4 sind auch als Sequenz denkbar). Die Debatte über diese unterschiedlichen Pfade wird, insbesondere in der US-amerikanischen Diskussion, intensiv geführt (Brzezinski 1998; Huntington 1999; Kupchan 1999; Haas 1999; Rieff 1999; deutsche Debatte: Nuscheler 1998; Zürn 1998; Messner/Nuscheler 1999). Dabei stehen drei miteinander verknüpfte Fragenkomplexe, die in der Literatur sehr kontrovers diskutiert werden, im Rahmen dieses Aufsatzes jedoch nicht weiter vertieft werden können, im Zentrum (Mürle 1998): (1) Welche dieser Szenarien sind aufgrund der Interessenlagen, Verteilung von Machtressourcen, der Handlungsfähigkeit jeweiliger Akteure sowie der Eigendyanmiken und Pfadabhängigkeit ökonomischer und institutioneller Entwicklung wahrscheinlich? (2) Welche der Optionen wäre den Problemen, die im Prozeß der Glo- 
balisierung entstehen, angemessen und welcher Typus von Global Governance könnte vor diesem Hintergrund zu globaler politischer Stabilität, Friedenssicherung und einer Sicherung des Primates der Politik vor dem der Ökonomie beitragen? (3) Welches Konzept entspricht den normativen Anforderungen demokratischer Legitimation, universeller Menschenrechte sowie sozialer Gerechtigkeit und welche Bedeutung spielen normative Entwürfe und Leitbilder für reale Entwicklungsprozesse?

\section{Wie steht es um die Zukunft der Nationalstaaten?}

Die Nationalstaaten, die während der vergangenen zwei Jahrhunderte Zentren ihrer Gesellschaften und Hauptakteure der internationalen Politik waren, müssen nicht zu den Verlierern der Globalisierung werden, ihre Rolle wird sich jedoch nachhaltig verändern. Entwicklungspfade für Nationalstaaten ergeben sich im Spannungsfeld der folgenden Einflußfaktoren: Erstens bauen funktionsfähige übernationale Regulierungssysteme auf handlungsfähigen nationalen Institutionen auf, so daß die Globalisierung nicht zum Verschwinden, sondern zur Transformation nationalstaatlicher Politik beiträgt. Ohne leistungsfähige Nationalstaaten kann auch keine tragfähige Global Governance- Architektur entstehen. Zweitens führt die institutionelle Inter- oder gar Supranationalisierung (z.B. im Rahmen der EU, der WTO) zu einer immer intensiveren Einbindung der Nationalstaaten in grenzüberschreitende politische und institutionelle Mehrebenensysteme. In diesem Prozeß wird in der Tendenz die Exekutive gegenüber der nationalen Gesellschaft, ihren Interessengruppen, aber auch dem Parlament gestärkt. Regierungen können einen zunehmenden Teil ihrer Innenpolitik mit (realen, scheinbaren oder fiktiven) internationalen Mandaten, Sach- und Handlungszwängen begründen, die für zivilgesellschaftliche Akteure immer undurchschaubarer werden, und so die Chancen ihrer Durchsetzung verbessern. Nationalstaaten gewinnen also gegenüber ihren Gesellschaften an Handlungsspielraum (Streeck 1998), der im Zuge der Ausdifferenzierung der Innenpolitik geschrumpft war. Drittens setzt die wirtschaftliche Globalisierung die Nationalstaaten einem Wettbewerb um mobile Produktionskapazitäten aus und schwächt sie damit tendenziell gegenüber global mobilen Unternehmen und Kapitalbesitzern (Scharpf 1999: 35ff.). ${ }^{3}$ Streeck interpretiert diese potentiellen exit-Optionen mobiler Akteure gegenüber dem Staat - in Anlehnung an separatistische Bewegungen, die die territoriale Abspaltung von Nationalstaaten betreiben - als einen Trend in

3 Wichtig ist der Hinweis, daß die Unternehmen zwar über eine potentielle exit option verfügen, jedoch zugleich auch auf eine Vielzahl gesellschaftlicher Vorleistungen angewiesen sind und bleiben, die geographisch gebunden sind (Technologienetzwerke, Zulieferer-Cluster, Ausbildungssysteme, qualifizierte und spezialisierte Mitarbeiterpools usw.). Auch in der globalisierten Welt werden Unternehmen daher nicht zu rastlosen „Standort-Surfern“ (Eßer et a. 1996, Messner 1998,). 
Richtung eines „sektoralen Separatismus“ (Streeck 1998: 36), der die Gefahr in sich tragen könnte, daß die Gesellschaften in zahllose „unverbundene Themengemeinden" (Offe 1999: 14) zerfallen. Darüber hinaus steht die territorial begrenzte Reichweite regulativer Politik in einem eklatanten Mißverhältnis sowohl zu der transnational organisierten Ökonomie als auch zu den grenzüberschreitenden und Weltproblemen in einer Vielzahl anderer Politikfelder. Es sind diese Kernelemente, die auf die unterentwickelte Reichweite nationalstaatlicher Politik sowie die Notwendigkeit des „Regierens jenseits des Nationalstaates" (Zürn 1998) in der Epoche des Globalismus verweisen. Viertens beeinflussen grenzüberschreitende Prozesse und Dynamiken die Steuerungsfähigkeit der Nationalstaaten trotz dieser generell wirkenden Trends sehr unterschiedlich. So zeigt sich z.B. bei der Wirtschaftspolitik, daß große nationale Handlungsspielräume im Bereich der Technologie- und Innovationspolitik bestehen, während in den Feldern Geld- Währungs- und Finanzpolitik internationale Kooperation, Regulierung und z.T. gar Harmonisierung immer wichtiger werden, um globale Instabilitäten zu verhindern. Auch in anderen Politikfeldern gilt, daß die Reichweite der Nationalstaaten je nach Problemkonstellation stark divergiert, jedoch nicht linear abnimmt (Hauchler et al. 1999; Gummet 1996). Fünftens geraten die Nationalstaaten in Konkurrenz zu allen möglichen Akteuren, seien es NGOs, Unternehmen oder auch religiöse Gruppen. Jean-Marie Guéhenno (1999: 11) macht darauf aufmerksam, daß Nationalstaaten in diesem Prozeß als besondere, über allen anderen Akteuren stehende Identifikations- und Orientierungspunkte an Bedeutung verlieren. Sechstens stellt sich freilich auch heraus, daß Globalisierung nicht nur einen Motor zur Reduzierung nationalstaatlicher Handlungspotentiale darstellt, sondern auch Anreizstrukturen zur Folge haben kann, die Demokratisierung, Dezentralisierung, zwischenstaatliche Kooperation und eine höhere Effektivität und Effizienz staatlichen Handelns befördern (Fuhr 1998):

- Nationalstaaten werden international rechenschaftspflichtig: Neue internationale Rechtssetzungen, Institutionen und Regime schränken die nationalstaatliche Dominanz in einigen wesentlichen Bereichen (Menschenrechte, Umwelt, Sozialstandards) ein und etablieren weltweit Mindeststandards. Prozesse internationaler Verrechtlichung (z.B. die Einrichtung des Internationalen Strafgerichtshofes) führen dazu, daß die Legitimität einer Regierung zunehmend anhand internationaler Standards gemessen wird.

- Demokratie und die Zivilisierung von Marktwirtschaft kann „von außen“ wirkungsvoll unterstützt werden: Internationale NGOs mischen sich in der Menschenrechts-, Umwelt-, Entwicklungs- und Sozialpolitik verstärkt in „nationale" Politikprozesse ein.

- Nationalstaaten kommen im Globalisierungsprozeß unter konstruktiven Effektivitäts- und Effizienzdruck: Staaten werden weltweit mit neuen sozialen Gruppen konfrontiert, die mit Verweis auf internationale Abkommen, Kon- 
ventionen und Erfahrungen (z.B. im Agenda 21-Prozeß) eine bessere Qualität staatlicher Dienstleistungen, funktionsfähige Regulierungssysteme und Partizipation einfordern.

Die ökonomische Globalisierung stärkt Dezentralisierungsprozesse in den Nationalstaaten und regionale Kooperation in den Weltregionen: Zentralregierungen geraten im Kontext der Weltwirtschaft unter den Druck ihrer Regionen, die ihr Profil im globalen Wettbewerb schärfen wollen. Insbesondere in Entwicklungsländern öffnen sich damit Chancen, die Politik näher an die Bevölkerung heranzubringen. Zugleich beschleunigt die Globalisierung Prozesse regionaler ökonomischer und politischer Kooperation in allen Weltregionen, die in der Vergangenheit immer wieder an nationalen Engstirnigkeiten gescheitert war. Erfahrungen aus der OECD-Welt zeigen, daß wirtschaftliche und politische Verflechtung zur Schaffung von Friedenszonen beitragen können.

\section{Zur globalen Kooperation verdammt?}

Ist Global Governance und zunehmende Kooperation zur Lösung globaler Probleme im Kontext des „Wettbewerbs der Staaten“ überhaupt eine realistische Perspektive? Und welche Formen nimmt die „Macht“, das „Medium aller Politik“ in der Ära des Globalismus an? Diese Fragen sind zentral für jede Diskussion über die Zukunft der Politik.

\section{Die Globalisierung stellt die Logik der internationalen und globalen Politik auf den Kopf}

Wolfgang H. Reinicke hat in seinen Überlegungen zu Elementen einer „Global Public Policy“ überzeugend die qualitativen Veränderungen, welche die Globalisierung für die Architektur und Dynamik der Politik bedeuten, herausgearbeitet. Dies gelingt ihm vor dem Hintergrund einer Differenzierung zwischen „interner“ und „externer Souveränität“ sowie einer präzisen Beschreibung des Übergangs von einem durch „komplexe Interdependenzen“ (Keohane/Nye 1977) charakterisierten Internationalen Systems, zur Logik der Globalisierung (Reinicke 1998: 52-74). Interne und externe Souveränität beschreibt Reinicke als komplementäre Konzepte. Externe Souveränität bezieht sich auf die Relationen zwischen Staaten im Internationalen System; interne Souveränität umschreibt die Beziehungen des Staates, mit gesellschaftlichen Akteuren und der Wirtschaft innerhalb des nationalen Territoriums: Mit bezug auf Max Weber formuliert er, daß

„internal sovereignty refers to the formulation, implementation, and maintenance of a legal, economic, political, and social order ... Internal Sovereignty came to describe the relationship between ... government and society ... In operational terms, internal sovereignty ... means the ability of a government to formulate, implement, and manage public policy. ... A threat to a country's operational internal sovereignty implies a threat to ist ability to conduct public policy.“ (Reinicke 1998: 56-57) 
Das Konzept der externen Souveränität bezieht sich auf das Verhältnis zwischen Staaten in einem internationalen System, das keine Zentralinstanz und kein Machtmonopol kennt und daher durch Anarchie gekennzeichnet ist. Staaten sind in diesem Kontext darauf ausgerichtet, ihre „Independenz“ (Unabhängigkeit), also ihre externe Souveränität, so weit wie möglich zu wahren und für ihre Sicherheit zu sorgen.

Die Perzeption zunehmender ökonomischer, politischer, sozialer und militärischer Vernetzungen zwischen den Staaten, führte in den Theorien der Internationalen Beziehungen seit den 70er Jahren zur Diskussion über das Konzept der „Interdependenz“ zwischen Staaten (Kaiser 1970; Keohane/Nye 1977). Dabei wird Interdependenz in der Regel als eine „relationship costly to break" verstanden (Waltz 1970). Robert Keohane und Joseph Nye (1977) haben mit ihrem Begriff der „komplexen Interdependenz“ auf einen qualitativen Wandel im Internationalen System hingewiesen. Die formal „independenten" Nationalstaaten sind durch eine zunehmende Zahl von Kanälen miteinander verbunden und daher wechselseitig immer stärker voneinander abhängig (interdependent) und verwundbar, insbesondere in bezug auf das Sicherheitsdilemma in einer anarchischen Welt ohne Gewaltmonopol. Die zentralen Elemente der Debatte um „komplexe Interdependenz“ sind demnach Nationalstaaten (als die beobachteten Akteure), sowie „externe“ Faktoren (die die Vulnerabilität der Staaten und ihre Interdependenz steigern). Die Zunahme „komplexer Interdependenz“ im Internationalen System stellt aus dieser Perspektive eine Herausforderung für die externe Souveränität der $\mathrm{Na}-$ tionalstaaten dar.

Die Folgen zunehmender Interdependenz werden von den Schulen der Internationaler Beziehungen unterschiedlicher eingeschätzt. Aus Sicht der realistischen und im Kern auch der neorealistischen Schule (Waltz 1979) läßt sich das Verhalten der Nationalstaaten und die Herausbildung „nationaler Interessen" aus der Struktur des Internationalen Systems ableiten, dessen Dynamik - auch unter der Bedingung steigender Interdependenzen - vor allem durch die Abwesenheit einer zentralen Instanz bestimmt wird. Die Anarchie des Internationalen Systems übersetzt sich in Wettbewerb und Konflikthaftigkeit der zwischenstaatlichen Beziehungen und begrenzen die Kooperationsfähigkeit der Staaten sowie die Chance, gemeinsame Interessen zu erkennen, strukturell. Im Ergebnis können die Staaten ihre externe Souveränität nur durch „adversarial competition“ (Reincke 1998: 61) verteidigen. Die liberale Schule (Oye 1986; Czempiel 1993) teilt die Einschätzung, das Internationale System sei durch Anarchie und daher den Wettbewerb der Staaten gekennzeichnet. Die Autoren betonen jedoch, daß - insbesondere aufgrund „komplexer Interdependenz“ - im Prozeß der sich verdichtenden Interaktion der Staaten unter spezifischen Umständen Kooperation und reziproke Beziehungen entstehen können (Axelrod 1984). „Cooperative Competition“ (Rei- 
nicke 1998: 61) ist hier der Schlüssel zur Bewältigung internationaler Interdependenzbeziehungen und zur Sicherung externer Souveränität. ${ }^{4}$

Die Globalisierung, die Reinicke primär ökonomisch (und damit aus meiner Perspektive (Messner 1999a) verengt, jedoch hinsichtlich der zentralen Entwicklungsdynamik zutreffend) perzipiert, geht über das Muster „komplexer Interdependenz" hinaus. Die Betonung liegt hier erstens nicht auf der Verdichtung zwischen(inter-)staatlicher Beziehungen, sondern auf „cross-border Strukturen“, die dazu führen, daß nationale und „externe“ Strukturen sich überkreuzen und vermischen und damit die klare Trennung zwischen Innen und Außen erodiert. Zweitens, ,as an economic dynamic, ..., globalization differs from interdependence in that it subsumes or internalize into its own institutional structure economic activities that previously took place between national markets, that is, between distinct economic and political units" (Reinicke 1998: 63, Hervorh. D.M.). Die Globalisierung integriert also ökonomische Räume und entkoppelt sie damit - nicht vollständig aber in Teilbereichen - von der Reichweite der nationalstaatlichen Politik. Dieser Prozeß schwächt die interne Souveränität der Staaten, zwar nicht im legalen, rechtlichen, jedoch im operationalen Sinne. Während es im Rahmen „komplexer Interdependenz" um externe Souveränität, also das Management zwischenstaatlicher Beziehungen und vor allem die Herstellung von Sicherheit im anarchischen Internationalen System ging, sind die Nationalstaaten in der Epoche des Globalismus darüber hinaus in einer zunehmenden Zahl von Politikfeldern (z.B. der Steuer-, der Sozial-, der Umweltpolitik) nicht mehr dazu in der Lage, Probleme in ihren nationalstaatlichen Grenzen im Alleingang zu lösen, da zentrale Steuerungsressourcen außerhalb der Grenzen verteilt sind und der Standortwettbewerb die Handlungsoptionen nationaler Regierungen begrenzt.

Aus dieser Perspektive läßt sich mit Verweis auf das zentrale Eigeninteresse der Staaten (und nicht „nur“ - wie sonst üblich - durch Insistieren auf zunehmend globalen Problemkonstellationen) ein überzeugendes Plädoyer für Global Governance ableiten. Die „geteilten (internen) Souveränitäten“ der Nationalstaaten (Messner/Nuscheler 1997; Messner 1998) müssen gepoolt werden, da nur so die Handlungsfähigkeit der Politik und deren interne Souveränität wiederhergestellt werden können. Um in diese Richtung zu agieren, müssen die Nationalstaaten in den zwischenstaatlichen Beziehungen, den multi- sowie supranationalen Organisationen sowie in Interaktion mit der Gesellschaftswelt Kooperationen aufbauen, „on a scale and depth not yet witnessed“ (Reinicke 1998: 70) - nur so kann die Erosion interner Souveräni-

4 Wichtig ist der Hinweis, daß Interdependenzen so groß sein können, daß sie Akteure „verwunden“; sie können aber auch so leicht sein, daß sie von ihm nur „empfunden“ werden (Czempiel 1999: 46). Es existieren zudem symmetrische Interdependenzverhältnisse, die Kooperation unmittelbar begünstigen und asymmetrische Interdependenzmuster, die Kooperation erschweren (Messner 1998: 217f.). 
tät der Staaten gestoppt werden. Die Frage nach der internen Souveränität der Staaten wird somit zu einem Gegenstand der Weiterentwicklung und Ausgestaltung globaler Politik. Damit stellt sich, ähnlich wie in der Debatte über komplexe Interdependenz, erneut die Frage nach der Chance für kooperative Beziehungen in der Ära des Globalismus bzw. im Kontext der Global Governance-Architektur.

\section{Vom Ordnungsmuster der "cooperative competition" zur "competitive cooperation"}

Entscheidend ist nun, daß sich die Grundmuster der internationalen Politik beim Übergang vom Regime der Interdependenz zur Globalisierung fundamental verändern. Unter den Bedingungen der Interdependenz waren sich Realisten und Liberale einig, daß die Anarchie des Internationalen Systems den Wettbewerb der Staaten als Mittel zur Verteidigung ihrer externen Souveränität fördert. Während die (Neo-)Realisten ,adversarial competition“, also dauerhafte Instabilität und Konfliktträchtigkeit infolge der Versuche der Staaten ihrer Handlungsspielräume auf Kosten anderer zu erweitern, für wahrscheinlich hielten, sahen die Liberalen immerhin Spielräume für „,cooperative competition“. Unter den Bedingungen der Globalisierung ist die Sicherung interner Souveränität jedoch nicht in einem konfliktiven Wettbewerb der Nationalstaaten (adversarial competition) zu sichern, sondern nur durch Kooperation zwischen ihnen (und Akteuren der Gesellschaftswelt). Die Fähigkeit zur Kooperation im internationalen System bzw. der Global GovernanceArchitektur wird so grundlegend für die Fähigkeit der Nationalstaaten, ihre interne und externe Souveränität und Handlungsfähigkeit, Legitimation und Identität zu reproduzieren. Damit wird die klassische Kernaussage der Theorien der Internationalen Beziehungen vom Kopf auf die Füße gestellt:

„Just as competition was rational for state engagement in the international system under interdependence, so the need to cooperate motivates states' behavior (präziser wäre: „should motivate", D.M.) under conditions of globalization. ... The same reasoning that led neorealists to characterize the international system as competitive (internal sovereignty cannot be achieved under conditions of anarchy at the domestic level, and therefore anarchy must be exported') now leads to the conclusion that the international system should be cooperative, because one central dimension, a state's identity, can only be achieved collectively.“ (Reinicke 1998: 72)

Während „cooperative competition“ das Interaktionsmuster zwischenstaatlicher Beziehungen im Kontext komplexer Interdependenz auf den Begriff bringt, verkehrt sich die Formel zur politischen Bewältigung der Globalisierung aufgrund der skizzierten strukturellen Veränderungen in „competitive cooperation“. Hierbei bezieht sich der Wettbewerb nicht auf den Output der Interaktion bzw. das grundlegende Ordnungsmuster, sondern auf die Methode bzw. den Prozeß, der eine kooperative Ordnung begründet. „Wettbewerb“ unter den Bedingungen von „competitive cooperation“ muß also verstanden 
werden als ein gemeinsamer Verhandlungs-, Such- und Lernprozeß von Akteuren mit divergierenden sowie zugleich interdependenten Interessen, in dessen Verlauf durchaus unterschiedliche Problemlösungsvorstellungen eingehen und ausprobiert werden (Wettbewerb der Ideen), der letztlich aber auf kooperativen Übereinkünften und Regimen aufbaut, die die Sicherung der internen Souveränität der Staaten und die Bearbeitung globaler Probleme ermöglichen.

Reinicke ist nicht so naiv, die Herausbildung einer kooperativen globalen Ordnung für einen Automatismus zu halten (Reinicke 1998: 1), aber er liefert eine exzellente Begründung für den notwendigen Abschied von einer hegemonialen und auf die unilaterale Durchsetzung „nationaler Interessen“ gegenüber anderen Staaten ausgerichteten Strategie der Außenpolitik. Diese Begründung sollte auch überzeugte (Neo-)Realisten (mit Weitblick) zu einer kritischen Überprüfung ihrer Argumentation motivieren - und zwar insbesondere deshalb, weil Reinicke ihre strukturellen Argumentationsfiguren auf die neuen Realitäten anwendet und hierbei zu einem Ergebnis kommt, daß den klassischen Kernaussagen der (Neo-)Realisten diametral entgegensteht. Natürlich kann Kooperation scheitern. Aber die skizzierte Entwicklungsdynamik der Globalisierung erzwingt geradezu kooperative Interaktionsmuster in der Global Governance-Architektur, erstens weil konfliktive Strategien in einer zunehmenden Zahl von Problemfeldern definitiv zum Scheitern verurteilt sind und zweitens weil die umfassende Erosion staatlicher Souveränität droht. ${ }^{5}$

\section{Wie weit oder eng sind die Möglichkeitsräume für globale Kooperation?}

Das strukturelle Grundmuster in der internationalen und globalen Politik begünstigt kooperative Handlungsstrategien. Damit ist allerdings noch nichts über die Möglichkeitsräume, also die Felder, in denen Global Governance Aussicht auf Erfolg hat, gesagt. Auf vier in diesem Kontext wichtige Merkposten möchte ich hinweisen.

Erstens muß berücksichtigt werden, daß Global Governance - aufgrund des Fehlens einer übergeordneten Hierarchie in der globalen Politik - in grenzüberschreitenden Verhandlungssystemen stattfindet. In diesen Netzwerkstrukturen können Koordinationsprobleme auftauchen, die den Möglichkeitsraum der Politik systematisch begrenzen (Messner 1998: 226-231). Zwar kann mit

5 Es ist zumindest interessant, daß der hartgesottener Realist Samuel Huntington in einem im Sommer 1999 veröffentlichten Aufsatz darauf aufmerksam gemacht hat, daß die USA lernen müssen, sich kooperativ in der globalen Politik zu verhalten, um auf mittlere Sicht nicht an Handlungsfähigkeit zu verlieren (Huntington 1999). In diese Richtung argumentiert auch R. Haass (1999: 38), stellvertretender Direktor der Brookings Institution: „The proper goal for American foreign policy is to encourage a multipolarity characterized by cooperation and concert rather than competition and conflict. ... such a world ... would be founded in a broader agreement on global purposes and problems. 
Verweis auf das Coase-Theorem und mit dem Instrumentarium der Spieltheorie gezeigt werden, daß alle pareto-optimalen Wohlfahrtsgewinne, die durch eine ideale hierarchische Koordination erreichbar wären, auch durch freiwillige Vereinbarungen zwischen autonomen, ausschließlich egoistisch-rationalen Akteuren realisiert werden können (Coase 1969). Für internationale Verhandlungssysteme läßt sich daher sagen, daß pareto-optimale Lösungen von Problemen theoretisch möglich sind. Damit ist jedoch zugleich auch eine Grenze globaler Netzwerksteuerung benannt. Denn das Pareto-Optimum ist dann erreicht, wenn keine Änderungen mehr denkbar sind, die den Nutzen mindestens eines Beteiligten erhöhen würde, ohne daß irgend ein anderer deshalb schlechter gestellt werden müßte. Dieser wohlfahrtstheoretische Indikator vernachlässigt bewußt Aspekte von Verteilungsgerechtigkeit und die Möglichkeit der Umverteilung von Besitzständen. Einen politisch interessanteren Wohlfahrtsmaßstab für die (global und nationale) Politik bietet das KaldorKriterium, das alle Maßnahmen positiv bewertet, deren Nutzen für die Begünstigten groß genug sind, um daraus die volle Entschädigung für diejenigen zu zahlen, die durch diese Maßnahmen benachteiligt werden. Im Zentrum des Kaldor-Konzepts steht also ein kollektiver Nutzen und Lösungen für ein Gesamtsystem - nicht nur individuelle Nutzenmaximierung, wie im Falle des Pareto-Optimums (Kaldor 1939). Eine hierarchische Entscheidungsinstanz kann sich über den Einspruch einzelner Betroffener hinwegsetzen und ist theoretisch in der Lage, Gemeinwohlmaximierung zu betreiben. In der Global Governance-Architektur existiert jedoch keine weltstaatliche Instanz. Der Versuch einer Annäherung an das Kaldor Optimum auf der Basis von internationalen Verhandlungssystemen stößt auf drei spezifische Probleme (Messner 1998: 228f.):

- Lösungen, die die aggregierte Wohlfahrt maximieren, werden systematisch ignoriert, wenn nicht konvergierende Interessenlagen vorliegen und die Lösungen nicht gleichzeitig auch für jeden der Netzwerkakteure eine Verbesserung gegenüber dem status quo implizieren.

- Für den Fall, daß unter diesen Bedingungen mehr als eine pareto-superiore Lösung möglich sein sollte, besteht die Gefahr, daß die Verhandlungen durch den Streit über die Wahl zwischen den Lösungen blockiert wird.

- Daraus folgt, daß Netzwerke nur dann in der Lage sind, das KaldorOptimum anzusteuern, wenn sie sich nicht von kompetitiven oder gar feindlichen Handlungsorientierungen gegenüber anderen Netzwerkteilnehmern (im Sinne von „dem anderen Schaden zufügen“) leiten lassen. Eine auf gemeinsame Problemlösung orientierte Handlungsstrategie ist Voraussetzung für erfolgversprechende Netzwerksteuerung.

Diese Problemkonstellationen lassen sich jedoch in einem Sinne modifizieren, der im Kontext globaler Politik durchaus denkbar und zuweilen üblich ist. Verändert man die skizzierten Voraussetzungen dahingehend, daß Aus- 
gleichszahlungen, mit denen die Verlierer entschädigt werden können, möglich sind und als Instrument eingesetzt werden (ein empirisches Beispiel wären die GEF-Mittel als Bestandteil globaler Klimapolitik), so sind Lösungen für die skizzierte Problematik denkbar. Eine wesentliche Herausforderung zwischen den jeweils beteiligten Akteuren wird darin bestehen, ein gemeinsames Verständnis von Interessenausgleich und Verteilungsgerechtigkeit herauszubilden (Messner 1998: 264ff.).

Zweitens funktioniert internationales Krisenmanagement bisher vor allem dann, wenn die Interessen wichtiger global player unmittelbar bedroht sind. Es können „schwarze Löcher“ im Global Governance-System entstehen, die sich aus stark asymmetrischen Interdependenzstrukturen ergeben: Die Stabilisierung der asiatischen Ökonomien ist für das Weltwirtschaftsgefüge und die internationalen Banken von großer Bedeutung; hier wurde rasch gehandelt und sehr viel Geld mobilisiert, um eine Ausbreitung der Krise zu verhindern. Andere drängende Zukunftsprobleme ganzer Weltregionen werden hingegen mit deutlich weniger Nachdruck angegangen: Die Verhandlungen über die Entschuldung der ärmsten Entwicklungsländer, in denen es um vergleichsweise geringe finanzielle Volumina geht, zogen sich beinahe zwei Dekaden hin; die Mittel für die Bekämpfung weltweiter Armut sinken tendenziell. Diese "selektiven Formen von Global Governance" sind nicht nur aus normativen Gründen der Fairneß fragwürdig; sie sind vor allem auch der Herausbildung einer umfassenden globalen Kooperationskultur in der Weltgesellschaft abträglich und unterminieren die Bereitschaft der schwächeren Länder zur Zusammenarbeit - und damit in längerer Perspektive die Chancen auf die dauerhafte Bewältigung von Weltproblemen (z.B. im Umweltbereich), zu deren Lösung auch diese Gesellschaften beitragen müssen. Die skizzierten „blinden Flecken der internationalen Kooperation" verweisen auch auf die Grenzen einer Strategie ausschließlich deliberativer Politik im internationalen System, die einseitig auf die Selbstorganisation und langfristige Orientierung der global player vertraut (Fuhr 1998). So wie Nationalstaaten, kann sich auch die Weltgesellschaft nicht ausschließlich auf das ,good will“ der handlungsmächtigsten Akteure verlassen. Verbindliche Ordnungssysteme und Regelwerke waren eine wesentliche Grundlage der Zivilisierung von nationalen Gesellschaften; nichts spricht dafür, daß man in einer immer interdependenteren Weltgesellschaft auf sie verzichten könnte.

Drittens ist zu befürchten, daß die Solidaritätsfähigkeit der globalen Zivilgesellschaft sowie der handelnden Akteure innerhalb der Global GovernanceArchitektur hinter der Mobilisierungsfähigkeit von Solidarität in den Nationalstaaten zurückbleiben wird - mit anderen Worten also redistributive Politiken in der globalen Politik unwahrscheinlicher sind, als auf nationaler Ebene. Diese Vermutung basiert auf der Überlegung, daß nationale Umverteilungspolitiken stark an kollektive Wertegemeinschaften rückgebunden sind. 
Während staatsbürgerliche Solidarität auf spezifischen, lange gewachsenen kollektiven Identitäten aufbauen kann, die Michael Walzer (1996) mit dem Begriff der „dicken Moral“ umschreibt, muß sich weltbürgerliche Identität im günstigsten Falle auf den in den Menschenrechten ausgedrückten moralischen Universalismus allein stützen, den Walzer als „,dünne Moral“ charakterisiert. Es ist anzunehmen, daß die „dicke Moral“ die Grundlage aktiver Solidarität zwischen Staatsbürgern ist, die redistributive Politiken im Rahmen der Wohlfahrtsstaaten zumutbar macht. Die universalistische, aber zugleich „dünne Moral“ der Weltbürger ruft zwar Affekte der Empörung über staatliche Repression und massive Menschenrechtsverletzungen hervor, es ist aber nicht ausgemacht, ob sie auch redistributive Politiken im Weltmaßstab trägt, die in der Lage wären, die großen Entwicklungsunterschiede zwischen den Nationen spürbar zu reduzieren oder die ökologischen Verteilungskonflikte der Zukunft zu entschärfen. Daraus ergeben sich drei Schlußfolgerungen: Erstens muß der internationale Menschenrechtsdiskurs durch Dialoge über globale (Verteilungs-)Gerechtigkeit ergänzt werden. Zweitens sind politische Initiativen bedeutend, um die Erosion „dicker Moral“ im nationalstaatlichen Kontext zu verhindern. Drittens muß in der EU die Herausforderung angenommen werden, sukzessive kollektive Identitäten herauszubilden, die über den Universalismus einer „dünnen Moral“ hinausreichen und soziale Integration und Interessenausgleich in Europa ermöglichen.

Viertens könnte sich eine Verengung des Möglichkeitsraumes in der Global Governance-Architektur durch exklusive Partizipationsmuster ergeben. Etwas holzschnittartig argumentiert, können zwei Akteursgruppen unterschieden werden. Zum einen die gut qualifizierten Akteure, die sich in der globalen Ökonomie problemlos positionieren können, von den Liberalisierungen profitieren, auf die sozialen Sicherungssysteme der Nationalstaaten, die in allen OECD-Ländern unter Globalisierungsdruck kommen, nicht angewiesen sind und unter den Bedingungen der ökonomischen und institutionellen Globalisierung ihre Partizipationsansprüche noch über den nationalen in den globalen Raum verlängern können. Es sind nicht zuletzt die Angehörigen dieser Gruppe, die den Kern der global agierenden NGOs ausmachen. Zum anderen diejenigen, die eher zu den ökonomischen Verlierern der Globalisierung gehören und aus deren Perspektive die sozialen Institutionen der demokratischen Wohlfahrtsstaaten von steigender Bedeutung sind, weil Globalisierung größere soziale Unsicherheiten mit sich bringt. Doch gerade wegen der Standortkonkurrenz wird die „Verteidigung“ der Wohlfahrtssysteme immer schwieriger, zumindest solange die OECD-Länder untereinander nicht zu stärkerer Politikkoordination in der Lage sind. Die Chancen dieser Akteursgruppe, sich an Formen „kosmopolitischer Demokratie“ zu beteiligen, dürften begrenzt sein. Die entstehende globale Zivilgesellschaft könnte zu einem exklusiven Club werden. 
Gerade weil es zur Entwicklung einer tragfähigen Global GovernanceArchitektur in der Epoche des Globalismus keine überzeugende Alternative gibt, und Kritiken an diesem Suchprozeß, in denen das "technokratische“ Global Governance-Projekt „schlimmstenfalls ... (als) ein Beitrag zur Modernisierung des Neoliberalismus, bestenfalls (als eine) versöhnlerische Utopie“ karikiert werden (Brunnengräber/Stock 1999: 466), kaum weiterhelfen, müssen die skizzierten Problemfelder hinsichtlich der Möglichkeiten, sie produktiv zu bearbeiten, genauer überprüft werden.

\section{Formwandel der Macht oder Restauration klassischer Machtpolitik in der Global Governance-Architektur?}

Wie steht es um das klassische Machtinstrumentarium der Staaten unter den Bedingungen der Globalisierung? Der Verbleib der USA als einziger Supermacht, die im Verlauf der 90er Jahre zu einem unverblümten Unilateralismus tendierte (z.B. im Verlauf der Einrichtung des Internationalen Strafgerichtshofes, der Aufkündigung des Atomwaffenteststops, der Verweigerung der Ratifizierung des Vertrages zum Verbot der Herstellung von Anti-Personen-Minen, der Schwächung der Institutionen der Vereinten Nationen) nach der Devise „soviel Unilateralismus wie möglich, soviel Multilateralismus wie unbedingt nötig“" (Nuscheler 1999: 11) sowie die Diskussion um den Einsatz von militärischer Gewalt zum Schutz von Menschenrechten, die auch unter dem Rubrum einer „Remilitarisierung der Außenpolitik“ geführt wird, sind für einige Autoren Anlässe, um vor einer „Rückkehr (der Weltpolitik) zu den Anfängen vor 1914“ zu warnen (Schwarz 1995). Die Mehrzahl der professionellen Beobachter der Weltpolitik ist sich einig, daß ein Abdriften der Weltpolitik in Richtung einer „Superpower Governance“ (Nuscheler 1999) oder eines „liberalen Imperialismus“ (Rieff 1999) nicht nur ein höchst gefährliches, weil konfliktives Szenario der Globalen Politik impliziert, sondern darüber hinaus eine Rückkehr zu traditionellen Konzepten der Machtpolitik unter den Bedingungen der Globalisierung in mittlerer Sicht ein zum Scheitern verurteiltes Projekt ist (Huntington 1999, Haass 1999, Czempiel 1999, Müller 1998, Messner/Nuscheler 1999). Die Globalisierung fordert einen Formwandel der Macht, denn am Anfang des 21. Jh. kann kein Nationalstaat, auch nicht die Supermacht USA, seine Ziele im Alleingang erreichen. All dies heißt nicht, daß Rückfälle in anachronistische hegemoniale Politikstrategien unmöglich wären. Vielmehr können sich zwischen den tradierten Perzeptions- und Handlungsroutinen der Nationalstaaten, die sich in den vergangenen Dekaden etabliert haben und den neuen Anforderungen an die Akteure der Weltpolitik, die durch die strukturellen Veränderungen der Globalisierung entstehen, „Anpassungslücken“ auftun - und zu Politikversagen führen (Seitz 1998). 
Czempiel verweist zu Recht darauf, daß die Macht das Medium aller Politik bleibt, denn „wer sie besitzt, kann seine Ziele erreichen“ (Czempiel 1999: 91). Doch was ist Macht? Wie steht es um die Ressourcen und erfolgversprechende Formen der Macht im 21. Jahrhundert?

Die Grenzen von „Zwang und Gehorsam": Der klassische Machtbegriff von Max Weber (1960: 42f.) betont die Fähigkeit, seinen Willen gegen den Widerstand anderer durchzusetzen und ,prompten, automatischen und schematischen Gehorsam bei einer angebbaren Vielfalt von Menschen zu finden." Dieser Machtbegriff verrät ein Politikmodell, daß noch der technischen Epoche der Mechanik entstammt und wird in einer global vernetzten Welt, in der schon aufgrund der Akteursvielfalt Selbststeuerung, Vetokräfte und nichtintendierte Folgewirkungen die Reichweite und Wahrscheinlichkeit gelungener unilateraler Durchsetzung von „Macht, Herrschaft und Gehorsam“ im Weberschen Sinne begrenzen. Man kommt einem Verständnis des Phänomens der Macht in den internationalen und globalen Beziehungen mit Rückgriff auf andere Autoren, die jeweils unterschiedliche Dimensionen der Macht ausleuchten, näher.

Macht als Überlegenheit: Robert Dahl (1956) beschreibt Macht als die Fähigkeit, einen Akteur zu einer Handlung zu veranlassen, die er sonst nicht unternommen haben würde. In diesem Machtbegriff bleibt der Kern von „Macht“, nämlich „Überlegenheit“, erhalten, und er läßt zugleich offen, wie sich diese Überlegenheit durchsetzt. Diese Definition erlaubt die Frage nach den sich wandelnden Ressourcen und Formen von Macht unter den Bedingungen sozio-ökonomischen Wandels.

Macht als Ressource ihrer eigenen Erosion: Karl Deutsch (1963: 21) verweist mit seiner Machtdefinition auf den ambivalenten und immer auch von Erosion bedrohten Charakter von Macht, wenn er schreibt, daß „Macht“ verstanden werden müsse als „die Fähigkeit, nicht lernen zu müssen.“ Einerseits erlaubt die Verfügbarkeit über Macht, nicht lernen zu müssen, da kein oder nur geringer Anpassungsdruck von seiten anderer Akteure besteht. Wer dächte vor diesem Hintergrund nicht an die „einzig verbliebene Supermacht USA“ am Anfang des 21. Jahrhunderts. Andererseits sind Machtpositionen stets prekär; insbesondere in Entwicklungssequenzen, in denen „lernen“ zu den wichtigsten Aktivposten zählt und die Verfügbarkeit über Informationen und Wissen (also die Ergebnisse von Lernprozessen) eine zentrale Machtressource darstellen. Die Warnung von Huntington an die „einsame Supermacht USA“, rasch das Kooperieren zu lernen, um nicht in der Weltpolitik von morgen isoliert zu sein und an Handlungsfähigkeit zu verlieren, läßt sich in diesem Rahmen interpretieren.

Grenzen der Gewalt und Bedeutsamkeit freiwilliger Folgebereitschaft: Von Karl Deutsch (1968) kann man auch etwas über die begrenzte Reichweite von Gewalt als Machtressource lernen. Er beschreibt Politik (auch die internatio- 
nale Politik) als ein Feld, in welchem Herrschaft und „gewohnheitsmäßige Folgsamkeit" einander überlappen. Aus dieser Überlegung leitet er einen Doppelcharakter der Macht ab: Herrschaft läßt sich in der Regel eher durch zentralisierte Organisationen ausüben; das Gleiche gilt für die Drohung mit Gewalt zur Erzwingung von „Folgebereitschaft“. Aber die verläßliche Folgebereitschaft von Akteuren kann kaum durch eine Hierarchie erzwungen oder organisiert werden. Folgebereitschaft und Grundlagen für konstruktive $\mathrm{Zu}$ sammenarbeit entstehen durch Überzeugung, Routinen und (scheinbar) fehlende Alternativen. „Der zentralisierte Gebrauch von Drohungen oder Gewalt ist daher kaum geeignet, ein dauerhaftes Ensemble politisch relevanter Gewohnheiten hervorzubringen" (ebd.: 33). Da gesellschaftliche Regeln nur dann einen Wert haben, wenn sie von 90\% der Akteure befolgt werden, weil sonst die Kosten $\mathrm{zu}$ deren Durchsetzung ins Unendliche steigen, folgert Deutsch (1968: 30), daß die freiwillige und gewohnheitsmäßige Folgebereitschaft der großen Mehrzahl der Akteure die ,unsichtbare, aber sehr reale Grundlage der Macht“ einer jeden Regierung ist. Diese Argumente können einen Neo-Realisten in der Phase komplexer Interdependenz der internationalen Beziehungen kaum von dessen Leitbild von "adversarial competition“ abbringen; sie sind jedoch unter den Bedingungen von „competitive cooperation" in der Epoche des Globalismus überzeugend.

Macht der Mächtigen und Macht der Schwachen: Crozier/Friedberg (1979: 40) präsentieren einen Machtbegriff für eine Epoche, in der Macht und Herrschaft im Sinne Webers nur noch die Ausnahme darstellt. Sie verweisen darauf, daß „Macht eine gegenseitige, aber unausgewogene Beziehung“ ist, und beschreiben Macht als eine Tauschbeziehung, die auf Gegenseitigkeit beruht, in der aber die Tauschbedingungen einen (oder mehrere) der beteiligten Akteure begünstigen: „Es ist ein Kräfteverhältnis, aus dem der eine mehr herausholen kann als der andere, bei dem aber gleichfalls der eine dem anderen nie völlig ausgeliefert ist" (ebd.: 41). Entscheidend ist die Verfügbarkeit über strategische Ressourcen, die nicht oder nur schwer substituierbar sind. Die Autoren unterscheiden vier zentrale Machtquellen (ebd.: 50), die auch in der globalen Politik von Bedeutung sind: (1) die Beherrschung strategischen Sachwissens, die sich (2) aus der Kontrolle über Informationen und Kommunikationsquellen ergeben, die sich wiederum (3) aus dem Vorhandensein allgemeiner organisatorischer Regeln oder auch Wertemuster ableiten lassen; und darüber hinaus (4) Repression und Gewalt, die aus der Perspektive der Mächtigen bestrafenden Charakter haben können oder zur Beendigung gewaltsamer Konflikte taugen mögen, jedoch keine „Gestaltungsmacht" darstellen.

Wichtig ist die Erkenntnis, daß Macht kein absolutes, sondern ein relatives, veränderbares soziales Verhältnis darstellt. Die Studie von Frank Biermann (1998) zur „neuen Verhandlungsmacht der Entwicklungsländer“ in der Weltumweltpolitik greift eine reale Machtverschiebung in der globalen Politik auf. 
Weil eine Vielzahl von globalen Umweltproblemen, denen sich auch „mächtige“ Industrieländer nicht entziehen können, nur in Kooperation mit Entwicklungsländern zu lösen sind, verschieben sich Kräfteverhältnisse, die auch „spill over-Effekte“ für andere Politikfelder haben können.

Drei Formen der Macht: Die Überlegungen von Ernst-Otto Czempiel (1999: 93ff.) können die Argumentation zur sich wandelnden Rolle der Kooperation und der Herausbildung des Grundmusters der „competitive cooperation“ in der Global Governance-Architektur komplementär ergänzen. Czempiel unterscheidet drei Formen von Macht, die in der globalen Politik des 21. Jahrhunderts von Bedeutung sein werden. „Beziehungsmacht“, im Sinne der direkten Einwirkung auf einen Akteur, ist die klassische und auch weiterhin wichtige Form der Anwendung von Macht, die zur diplomatischen Routine gehört. Beziehungsmacht nimmt Einfluß, um zu überzeugen, Vorteile zu erzielen, Nachteile zu vermeiden und Allianzen zu schmieden. Wirkungsvoller ist die „Definitions- und Konsensmacht“, die nicht unzählige Einzelhandlungen, sondern Ergebnisse zu beeinflussen versucht, die nur durch kollektives Handeln erreicht werden können. Die Definitions- und Konsensmacht ist darauf ausgerichtet, zu klären und vorzugeben, was zentrale Probleme sind, in welche Richtung Lösungen gesucht werden müssen und wie Konflikte vermieden oder bearbeitet werden können. Sie schafft damit einen gemeinsamen Rahmen und Leitbilder, die die Situationsdeutung harmonisieren. In einer immer dichter vernetzten Welt, in der die Steuerungsressourcen zur Lösung gemeinsamer Probleme immer breiter gestreut sind, gewinnt diese Form der Macht stark an Bedeutung. Die Definitions- und Konsensmacht speist sich vor allem aus den ersten drei Machtquellen, auf die auch Crozier/Friedberg verweisen.

Oft wird die Definitions- und Konsensmacht aufgrund der Erosion interner und externer Souveränität von Nationalstaaten nur als kollektive Kontrolle von Handlungsergebnissen (z.B. der EU, des Mercosur) möglich sein. Joseph Nye (1990) hat für diesen Typus von Macht den Begriff der "soft power" geprägt, der treffend ist, um die „Definitions- und Konsensmacht“ von militärischer oder auf Gewaltandrohung basierender Macht zu unterscheiden und zugleich irreführend ist, weil es darum geht „knallharte Ergebnisse“ zu erzeugen (Czempiel 1999: 94). Diese Form der Machtausübung ist eine strategische Antwort auf die Grenzen der Machterzwingung (und der Gewalt), auf die Karl Deutsch (1963/1968) schon vor langer Zeit verwiesen hat und die in einer durch geteilte Souveränitäten gekennzeichneten Welt von stetig zunehmender Bedeutung sind.

Die entwickeltste, wirkungsvollste und zukunftsträchtigste Form der Macht ist die „strukurelle Macht“ oder besser die „Fähigkeit zur Strukturbildung“, mit dem Ziel, über Strukturen (also Institutionen und Regeln) die zukünftigen Handlungen anderer Akteure sowie deren Interaktionen über lange Zeit- 
räume zu beeinflussen, ohne deren Autonomie direkt in Frage zu stellen (Guzzini 1993). So werden die Akteure, denen es in den kommenden Jahren gelingt, durch Definitions- und Konsensmacht sowie die darauf aufbauende Fähigkeit zur Strukturbildung, die Reorganisation von Weltbank und IWF zu prägen und die Herausbildung neuer Institutionen zur Regulierung der globalen Finanzmärkte aktiv voranzutreiben, die zukünftige Ausrichtung der Weltökonomie signifikant beeinflussen können.

Macht basiert im 21. Jahrhundert also nicht mehr auf der Beherrschung großer Territorien und nur sekundär auf Gewaltandrohung oder der Höhe der Rüstungsetats, sondern primär auf der strategischen, organisatorischen, wissensbasierten und auf gemeinsame Problemlösung ausgerichteten Kompetenz, komplexe Interaktionen zu steuern, Kooperation zu organisieren und durch Strukturbildung die Richtung des Wandels aktiv und zielorientiert (mit) zu gestalten. Konflikte werden selbst in einer kooperativ ausgerichteten Global Governance-Architektur eher zunehmen, denn die „Nähe“, die durch grenzüberschreitende Interdependenzen und das System geteilter Souveränitäten erzeugt wird, sowie eine steigende Zahl von Weltproblemen wird auch die Anlässe für Konflikte vermehren. Doch wer seine Umwelt veranlassen will, etwas zu tun (sei es, um gemeinsame Probleme zu lösen oder eigene Interessen durchzusetzen), muß im Regelfall darauf achten, den Konsens der Betroffenen aufrechtzuerhalten. Die skizzierten Formen der Macht sind tragfähig, wenn es darum geht, Antworten auf den Übergang zum ordnungspolitischen Grundmuster der „competitive cooperation“ in der internationalen und globalen Politik zu finden. Und um zu der Ausgangsfrage nach dem Verhältnis von Innen- und Außenpolitik zurückzukommen: Die Modalitäten der Macht in der „Außenpolitik“ nähern sich analytisch betrachtet dem „Gebrauch“ und den Formen von Macht in der Innenpolitik (Dahrendorf 1992) immer stärker an. Gewalt verschwindet nicht, ist jedoch nur noch funktional, tauglich und legitim, um extreme Regelverstöße zu beenden (Gewalttaten und schwere Kriminalität in der Innenpolitik; schwere Menschenrechtsverletzungen in der Außenpolitik) - im Kontext der Globalisierung sind die politischen Akteure zur Kooperation oder zu einer zunehmenden Handlungsunfähigkeit verdammt.

Deutlich wird zudem, daß die Globalisierung die Grundmuster der Politik der kommenden Dekaden und damit auch die Bedeutung und die Ressourcen von Macht weitgehend verändert. Länder, die die neuen Konstellationen klug nutzen, werden zu den Gewinnern des Umbruchs gehören. Andere können mit den „Methoden von gestern“ durchaus noch eine Weile erfolgreich sein. Sie werden damit die Konflikthaftigkeit in der globalen Politik erhöhen sowie die Herausbildung einer den neuen Rahmenbedingungen angemessenen stabilen globalen Ordnung verlangsamen; sie werden jedoch auch zu den (relativen) Verlierern der Globalisierung gehören. Insofern stellt der 
Umbruch auch ein „window of opportunity“ für Veränderungen in der Hierarchie von Weltgesellschaft und Weltwirtschaft dar.

\section{Literatur}

Albert, Mathias/Lothar Brock (1999): The Complexity of Global Change: Conceptualizing the Regulation of „Global Structures“, Manuskript, Ffm.

Altvater, Elmar/Birgit Mahnkopf (1996): Grenzen der Globalisierung, Münster.

Altvater, Elmar et al. (Hrsg.) (1997): Vernetzt und verstrickt. Nicht-Regierungsorganisationen als gesellschaftliche Produktivkraft, Münster.

Andreae, Lisette/Karl Kaiser (1998): Die „Außenpolitik“ der Fachministerien, in: W.-D. Eberwein/K. Kaiser (Hrsg.): Deutschlands neue Außenpolitik. Band 4, München. S. 29-46.

Axelrod, Robert (1984) The Evolution of Cooperation, New York.

Beisheim, Marianne et al. (1999): Im Zeitalter der Globalisierung?, Baden-Baden.

Benner, Thorsten/Wolfgang H. Reinicke (1999): Politik im globalen Netz, in: Internationale Politik, Nr. 8, S. 25-32.

Biermann, Frank (1998): Weltumweltpolitik zwischen Nord und Süd. Die neue Verhandlungsmacht der Entwicklungsländer, Baden Baden.

Böhret, Carl/Göttrik Wewer (Hrsg.) (1993): Regieren im 21. Jahrhundert - zwischen Globalisierung und Regionalisierung, Opladen.

Brunnengräber, Achim/Christian Stock (1999): Global Governance. Ein neues Jahrhundertprojekt?, in: PROKLA 116, S. 445-468.

Brzezinski, Zbigniew (1998): Die einzige Weltmacht, Ffm.

Burton, John (1972): World Society, Cambridge.

Coase, Ronald (1969): The Problem of Social Costs, in: Journal of Law and Economics, Nr.3.

Crozier, Michel/E. Friedberg (1979): Macht und Organisation, Königstein.

Czempiel, Ernst-Otto (1993): Weltpolitik im Umbruch, 2. Neubearbeitete Auflage, München.

- (1999): Kluge Macht, Außenpolitik für das 21. Jahrhundert, München.

Chace, James/Nicholas Rizopoulos (1999): Towards a New Concert of Nations, in: World Policy Journal, Fall 1999, S. 2-10.

Dahl, Robert (1956): A Preface to Democratic Theory, Chicago.

Dahrendorf, Ralf (1992): Der moderne soziale Konflikt, Stuttgart.

Delbrück, Jost (1998): Von der Staatenordnung über die internationale Kooperation zur „supraterritorial oder global governance“, in: Ulrich Bartosch/ Jochen Wagner (Hrsg.): Weltinnenpolitik, München, S. 55-66.

Deutsch, Karl W. (1963): The Nerves of Government, New York.

- (1968): Analyse internationaler Beziehungen, Ffm.

Durán, Clemente R./Enrique Dussel Peters (Hrsg.) (1999): Dinámica Regional y Competitividad Industrial, Mexico D.F.

Eberwein, Wolf-Dieter/Karl Kaiser: Wissenschaft und außenpolitischer Entscheidungsprozeß, in: dies. (Hrsg.): Deutschlands neue Außenpolitik, Band 4, München. S. 1-11.

Friedrich Ebert Stiftung (Hrsg.) (1996): Globale Trends und internationale Zivilgesellschaft: oder: NGOisierung der Weltpolitik?, Bonn.

Eßer, Klaus (Hrsg.) (1996): Globaler Wettbewerb und nationaler Handlungsspielraum, Köln.

Forschungsgruppe Weltgesellschaft (1996): Identifizierung eines „Phantoms“, in: Politische Vierteljahresschrift, Nr. 37, S. 6-25.

Fuhr, Harald (1998): Konstruktiver Globalisierungsdruck?, in GTZ (Hrsg.): Globalisierung: Chancen und Herausforderungen für die Entwicklungszusammenarbeit, Eschborn, S. 65-77.

Guéhenno, Jean-Marie (1995): The End of the Nation-State, Minnesota.

- (1999): Die neue Machtfrage, in: Die Zeit, 16.Dezember 1999, S. 11-12.

Gummet, Philip (Hrsg.) (1996): Globalization and Public Policy, Cheltenham.

Guzzini, Stefano (1993): Structural Power: The Limits of Neo-Realist Power Analysis, in: International Organization, Nr. 3, S. 443-461.

Haas, Peter M. (1992): Knowledge, Power, and International Policy Coordination, Cambridge.

Haass, Richard N. (1999): What to Do With American Primacy? In: Foreign Affairs, Nr. 5, S.37-49. 
Hauchler, Ingomar/Dirk Messner/Franz Nuscheler (Hrsg.) (1999): Globale Trends 2000, Ffm.

Held, David et al. (1999): Global Transformation, Stanford.

Héritier, Adrienne/Christoph Knill/Susanne Mingers (1996): Ringing the Changes in Europe, Berlin.

Hewson, Martin/ T.J.Sinclair (Hrsg. (1999): Approaches to Global Governance Theory, New York.

Hirsch, Joachim (1995): Der nationale Wettbewerbsstaat, Berlin/ Amsterdam.

Huntington, Samuel P. (1999): The Lonely Superpower, in: Foreign Affairs, Nr. 2, S. 35-49.

Kaiser, Karl (1970): Interdependenz und Autonomie, in: K. Kaiser/R. Morgan (Hrsg.): Strukturwandel der Außenpolitik in Großbritannien und der Bundesrepublik, München, S.50-70.

Kaiser, Karl/ Bernhard May (1995): Weltwirtschaft und Interdependenz, in: Karl Kaiser/ HansPeter Schwarz (Hrsg.): Die neue Weltpolitik, Bonn, S. 396-402.

Kaldor, N. (1939): Welfare Propositions of Economics and Inter-Personal Comparison of Utility, in: The Economic Journal, Nr. 49.

Kenis, Peter/Volker Schneider (1991): Networks and Policy Analysis, in: Bernd Marin/ Renate Mayntz (Hrsg.): Policy Networks, Ffm.

Keohane, Robert O./Joseph S. Nye (1977): Power and Interdependence, Boston.

Kleinsteuber, Hans/Barbara Thomaß (1998): Perspektiven und Probleme der Politikvermittlung im Zeitalter von Globalisierung und medientechnischer Revolution, in: Ulrich Sarcinelli (Hrsg.): Politikvermittlung und Demokratie in der Mediengesellschaft, Bonn, S. 209-229.

Knodt, Michéle (1998): Auswärtiges Handeln der deutschen Länder, in: Wolf-Dieter Eberwein/ Karl Kaiser (1998): Deutschlands neue Außenpolitik, Band 4, München, S. 153-166.

Kupchan, Charles A. (1999): Life after Pax Americana, in: World Policy Journal, Fall, S. $20-27$.

Mann, Michael (1997): Hat die Globalisierung den Siegeszug des Nationalstaates beendet?, in: PROKLA 106, S. 13-142.

Manning, Bayless (1977): The Congress, the Executive, and Intermestic Affairs, in: Foreign Affairs, Nr. 2, S. 306-324.

Mayntz, Renate/Fritz W. Scharpf (1995): Gesellschaftliche Selbstregelung und politische Steuerung, Ffm.

Messner, Dirk (1998): Die Netzwerkgesellschaft, Wirtschaftliche Entwicklung und internationale Wettbewerbsfähigkeit als Probleme gesellschaftlicher Steuerung, Köln (2. Aufl.).

- (Hrsg.) (1998a): Die Zukunft des Staates und der Politik, Bonn.

- (1999): Nicht-Regierungsorganisationen in der (Welt-)Politik, in: Karl-Josef Kuschel et al. (Hrsg.): Ein Ethos für eine Welt, Ffm/ New York, S. 332-362.

- (1999a): Strukturen und Trends in der Weltgesellschaft, in: Ingomar Hauchler/Dirk Messner/Franz Nuscheler (Hrsg): Globale Trends 2000, Ffm., S. 45-76.

Messner, Dirk/Franz Nuscheler (Hrsg.) (1996): Weltkonferenzen und Weltberichte, Bonn.

- (1997): Global Governance. Herausforderungen an der Schwelle zum 21. Jahrhundert, in: Dieter Senghaas (Hrsg.): Frieden machen, Frankfurt, S. 337-362.

- (1999): Strukturen und Trends der Weltpolitik, in: Hauchler, I./Messner, D./Nuscheler F. (Hrsg.): Globale Trends 2000, Frankfurt, S. 371-198.

Methews, Jessica (1997): The Power Shift, in: Foreign Affairs, Nr. 1, S. 50-66.

Milward, Alan S. (1992): The European Rescue of the Nation-State, Berkeley.

Müller, Harald (1998): Das Zusammenleben der Kulturen, Frankfurt/M.

Mürle, Holger (1998): Global Governance. Literaturbericht und Forschungsfragen, INEF-Report 32, Duisburg.

Narr, Wolf-Dieter (1999): Gegenwart und Zukunft einer Illusion. „Rot-Grün“ und die Möglichkeiten gegenwärtiger Politik, in: PROKLA 116, S. 351-376.

Nuscheler, Franz (1998): Eine neue Weltpolitik. Multilateralismus statt Pax Americana, in: Internationale Politik, Nr. 11, S. 10-16.

Nye, Joseph S (1990): Soft Power, in: Foreign Affairs, Autumn, S. 153-166.

- (1999): Redefining the National Interest, in: Foreign Affairs, July/August, S. 22-35.

Offe, Claus (1999): Wenn das Vertrauen fehlt, in: Die Zeit, 9. Dezember 1999, S. 12-13.

Ohmaie, Kenichi (1995): The End of the Nation-State, New York.

Oye, Kenneth A. (1986): Cooperation under Anarchy, Princeton.

Ploetz, von H.-F. (1998): Der Auswärtige Dienst vor neuen Herausforderungen, in: W.-D. Eberwein/K. Kaiser (Hrsg.): Die neue deutsche Außenpolitik, Band 4, München. S. 59-74.

Reinicke, Wolfgang H. (1998): Global Public Policy, Washington. 
Rieff, David (1999): A New Age of Liberal Imperialism? In: World Policy Journal, Summer, S.1-10.

Rosenau, James N. (1997): Along the domestic-Foreign Frontiers, Cambridge.

Scharpf, Fritz W. (1972): Komplexität als Schranke der politischen Planung, in: Politische Vierteljahresschrift, Nr. 4, S. 168-192.

- (1987): Grenzen der institutionellen Reform, in: Jahrbuch zur Staats- und Verwaltungswissenschaft 1987, Baden-Baden, S. 111-151.

- (1999): Regieren in Europa. Effektiv und demokratisch?, Ffm.

Schellenhuber, Hans Joachim/ Volker Wenzel (1999): Earth System Analysis, Berlin 1998.

Schwarz, Hans-Peter (1995): Die neue Weltpolitik am Ende des 20. Jahrhunderts - Rückkehr zu den Anfängen vor 1914?, in: K. Kaiser/H.-P. Schwarz (Hrsg.): Die neue Weltpolitik, Bonn, S. 15-33.

Seitz, Konrad (1998): Der Wettlauf ins 21. Jahrhundert, Berlin.

Siwert-Probst, Judith (1998): Die klassischen außenpolitischen Institutionen, in: Wolf-Dieter Eberwein/Karl Kaiser (Hrsg.): Deutschlands neue Außenpolitik, Bd. 4, München. S. 13-28.

Stallings, Barbara (Hrsg.) (1995): Global Change, Regional Response, Cambridge.

Storper, Michael (1997). The Regional World. New York/ London.

Streeck, Wolfgang (1991): On the Institutional Conditions of Diversified Quality Production, in: Egon Matzner/Wolfgang Streeck (Hrsg.): Beyond Keynesianism, Worcester.

- (Hrsg.) (1998): Internationale Wirtschaft, nationale Demokratie, Ffm.

Waltz, Kenneth N. (1979): Theory of International Politics, Addison.

Walzer, Michael (1996): Lokale Kritik - globale Standards, Berlin.

Weber, Max (1960): Soziologische Grundbegriffe, Tübingen.

Werle, Raymund (1995): Staat und Standards, in: Renate Mayntz/ Fritz W. Scharpf (Hrsg.): Gesellschaftliche Selbstregelung und politische Steuerung, Ffmr, S. 266-298.

Worldbank (1998): Beyond the Washington Consensus. Institutions Matter, Washington.

Young, Oran R. (1999): Governance in World Affairs, New York.

Zürn, Michael (1998): Regieren jenseits des Nationalstaates, Ffm. 